Neural, Parallel, and Scientific Computations 29 (2021) No.1, 1-19

\title{
VIABLE SOLUTIONS OF LIPSCHITZIAN QUANTUM STOCHASTIC DIFFERENTIAL EQUATIONS
}

\author{
AKINWUMI TITILAYO O AND AYOOLA EZEKIEL O \\ Department of Mathematics and Computer Science, Elizade University \\ Ilara-Mokin, Ondo State, Nigeria. \\ Department of Mathematics, University of Ibadan, Ibadan, Oyo-State, Nigeria.
}

\begin{abstract}
We establish the existence of viable solutions of quantum stochastic differential equations (QSDE) within the framework of Hudson and Parthasarathy formulation of quantum stochastic calculus. The main results are established for QSDE whose coefficients are Lipschitzian and quasicompact. This work extends the Nagumo viability results for classical differential equations on finite dimensional Euclidean spaces. Viable solutions of QSDE in the present formulation take values in some closed subsets of the infinite dimensional locally convex spaces of non-commutative stochastic processes.
\end{abstract}

AMS (MOS) Subject Classification. (2020) 60H10, 60H20, 81S25

Keywords and Phrases. Lipschitzian, Quasi-compact, Fock Spaces, Tangent cones

\section{INTRODUCTION}

This work initiates the study of viable solutions of quantum stochastic differential equations within the framework of Hudson-Parthasarathy formulation of quantum stochastic calculus [15] and using the notations of previous works in $[5,6,7,8,9,11$, $13,14]$. Viability theory concerning solutions of differential equations is important since it addresses the question of dynamic adaptation of real life systems to environments defined by constraints. It is well known that when modelling physical systems that are susceptible to quantum noise, quantum stochastic differential equations (QSDEs) often arise.

In analogy to the classical context, quantum systems and other physical systems are sometimes subject to some constraints. The temporal evolution of such systems maybe modelled by means of a noncommutative stochastic calculus [HudsonParthasarathy] [15] that generalises the classical Ito stochastic calculus. To be able to take account of the temporal evolutions of the observables of the quantum systems subject to some constraints, a viability theory needs to be developed in parallel to

Received November 1, 2020

www. dynamicpublishers . org;

https://doi.org/10.46719/npsc20202911.
1061-5369 \$15.00 (C)Dynamic Publishers, Inc.

www .dynamicpublishers.com; 
the classical theory. In this paper, we exploit the notion of a viable solution of QSDE taking values in some closed subset of the space of quantum stochastic processes.

In the classical settings, existence of viable solutions of differential equations defined on Euclidean spaces have been well studied see [1, 2, 3, 17, 18, 19, 20]. However, the viability problem for quantum stochastic differential equation have not been well studied. The existence of solutions of quantum stochastic differential equations and inclusions in which the coefficients are Lipschitzian have been established in $[4,5,9,11,13]$. In these previous works, the solutions were established in the global space of the quantum processes without any constraint. Our present efforts are partly aimed at provoking extensive study of the viability theory with respect to diverse topologies on the space of quantum stochastic processes as itemized in [12].

In the present paper, viable solutions of quantum stochastic differential equations are established subject to the coefficients being Lipschitzian and quasi compact. This is done by restricting the solutions to some closed subset of the global locally convex space.

We employ the properties of some cones that are tangent to the set of matrix elements of a closed subset of the space of stochastic processes. This enables us to establish the existence of solutions of the QSDE whose matrix elements are viable with respect to the given closed set.

The organization of the paper is as follows. Section 2 highlights some of the fundamental notions and notations which are used throughout the discussion. Section 3 introduces the quantum stochastic differential equations under consideration. Section 4 describes the notion of viable solutions with respect to the weak topology on the space of the observables generated by a family of seminorms while section 5 is devoted to the main results of this paper.

\section{PRELIMINARIES}

Let $D$ be an inner product space and $H$, the completion of $D$. We denote by $L^{+}(D, H)$, the set $\left\{X: D \rightarrow H: X\right.$ is a linear map satisfying $D o m X^{*} \supseteq D$, where $X^{*}$ is the operator adjoint of $\left.X\right\}$.

We remark that $L^{+}(D, H)$ is a linear space under the usual notions of addition and scalar multiplication of operators.

In what follows, $\mathbb{D}$ is some inner product space with $R$ as its completion, and $\gamma$ is some fixed Hilbert space. 
For each $t \in \mathbb{R}_{+}$, we write $L_{\gamma}^{2}\left(\mathbb{R}_{+}\right)$,(resp. $L_{\gamma}^{2}([0, t))$ resp. $\left.L_{\gamma}^{2}([t, \infty))\right)$, for the Hilbert spaces of square integrable, $\gamma$-valued maps on $\mathbb{R}_{+} \equiv[0, \infty)$, (resp. $[0, t)$; resp. $[t, \infty))$. Then we introduce the following spaces:

(i) $\mathcal{A} \equiv L^{+}\left(\mathbb{D} \otimes \mathbb{E}, \mathcal{R} \otimes \Gamma\left(L_{\gamma}^{2}\left(\mathbb{R}_{+}\right)\right)\right.$.

(ii) $\mathcal{A}_{t} \equiv L^{+}\left(\mathbb{D} \underline{\otimes} \mathbb{E}_{t}, \mathcal{R} \otimes \Gamma\left(L_{\gamma}^{2}([0, t))\right) \otimes 1^{t}\right.$.

(iii) $\mathcal{A}^{t} \equiv 1_{t} \otimes L^{+}\left(\mathbb{D} \otimes \mathbb{E}^{t}, \mathcal{R} \otimes \Gamma\left(L_{\gamma}^{2}([t, \infty))\right), t>0\right.$

where $\underline{\otimes}$ denotes algebraic tensor product and $1_{t}$ (resp. $\left.1^{t}\right)$ denotes the identity map on $\mathcal{R} \otimes \Gamma\left(L_{\gamma}^{2}([0, t))\right)\left(\operatorname{resp} . \Gamma\left(L_{\gamma}^{2}([t, \infty))\right), t>0\right.$. We note that $\mathcal{A}^{t}$ and $\mathcal{A}_{t}, t>0$, may be naturally identified with subspaces of $\mathcal{A}$. For $\eta, \xi \in \mathbb{D} \underline{\otimes} \mathbb{E}$, define $\|\cdot\|_{\eta \xi}$ on $\mathcal{A}$ by $\|x\|_{\eta \xi}=|\langle\eta, x \xi\rangle|, x \in \mathcal{A}$. Then $\left\{\|\cdot\|_{\eta \xi}, \eta, \xi \in \mathbb{D} \underline{\otimes} \mathbb{E}\right\}$ is a family of seminorms on $\mathcal{A}$; we write $\tau_{w}$ for the locally convex Hausdorff topology on $\mathcal{A}$ determined by this family. We denote by $\tilde{\mathcal{A}}, \tilde{\mathcal{A}}_{t}$ and $\tilde{\mathcal{A}}^{t}$ the completions of the locally convex topological spaces $\left(\mathcal{A}, \tau_{w}\right),\left(\mathcal{A}_{t}, \tau_{w}\right)$ and $\left(\mathcal{A}^{t}, \tau_{w}\right), t>0$, respectively.

We remark that the net $\left\{\tilde{\mathcal{A}}_{t} ; t \in \mathbb{R}_{+}\right\}$is a filtration of $\tilde{\mathcal{A}}$. Denoting by $\operatorname{Clos}(\tilde{A})$, the family of closed subsets of the topological space $\tilde{A}$ we adopt the Hausdorff topology on $\operatorname{clos}(\widetilde{\mathcal{A}})$ as described in $[4,5,6,7,8,9,11,13,14]$.

Definition 2.1: Let $I \subseteq \mathbb{R}_{+}$,

(i) A map $X: I \rightarrow \tilde{\mathcal{A}}$ is called a stochastic process indexed by $I$.

(ii) A stochastic process $X$ is called adapted if $X(t) \in \tilde{\mathcal{A}}_{t}$ for each $t \in I$.

We denote by $\operatorname{Ad}(\tilde{\mathcal{A}})$ the set of all adapted stochastic processes indexed by $I$.

(iii) A member $X$ of $\operatorname{Ad}(\tilde{\mathcal{A}})$ is called

(a) weakly absolutely continuous if the map $t \rightarrow\langle\eta, X(t) \xi\rangle, t \in I$, is absolutely continuous for arbitrary $\eta, \xi \in \mathbb{D} \underline{\otimes} \mathbb{E}$. We denote this subset of $A d(\tilde{\mathcal{A}})$ by $\operatorname{Ad}(\tilde{\mathcal{A}})_{\text {wac }}$.

(b) locally absolutely $p$-integrable if $\|X(\cdot)\|_{\eta \xi}^{p}$ is Lebesgue measurable and integrable on $\left[t_{0}, t\right) \subseteq I$ for each $t \in I, p \in(0, \infty)$ and arbitrary $\eta, \xi \in \mathbb{D} \underline{\otimes} \mathbb{E}$. We denote this subset of $\operatorname{Ad}(\tilde{\mathcal{A}})$ by $L_{\text {loc }}^{p}(\tilde{\mathcal{A}})$.

In what follow, we adopt stochastic integration in the frameworks of [11, 15]. 


\section{QUANTUM STOCHASTIC DIFFERENTIAL EQUATIONS}

Definition 3.1: Let $E, F, G, H \in L_{l o c}^{2}(I \times \tilde{\mathcal{A}})$ and $\left(t_{0}, X_{0}\right)$ be a fixed point of $I \times \tilde{\mathcal{A}}$. Then a relation of the form

$$
\left.\begin{array}{l}
d X(t)=E(t, X(t)) d \Lambda_{\pi}(t)+F(t, X(t)) d A_{f}(t)+G(t, X(t)) d A_{g}^{+}(t)+H(t, X(t)) d t \\
X\left(t_{0}\right)=X_{0}
\end{array}\right\}
$$

almost all $t \in I$ is called a quantum stochastic differential equation (QSDE) with coefficients $E, F, G, H$ and initial data $\left(t_{0}, X_{0}\right)$ if $X\left(t_{0}\right)=X_{0}$.

equation (3.1) is understood in integral form as:

$$
\left.\begin{array}{l}
X(t)=X_{0}+\int_{t_{0}}^{t}\left(E \left(s, X(s) d \Lambda_{\pi}(s)+F\left(s, X(s) d A_{f}(s)\right.\right.\right. \\
\left.+G(s, X(s)) d A_{g}^{+}(s)+H(s, X(s)) d s\right), \quad t \in I,
\end{array}\right\}
$$

with coefficients $E, F, G, H$ and initial data $\left(t_{0}, X_{0}\right)$.

An equivalent form of (3.1) has been established in Theorem 6.2 in [11], and is given by

$$
\left.\begin{array}{l}
\frac{d}{d t}\langle\eta, X(t) \xi\rangle=P(t, X(t))(\eta, \xi) \\
\left\langle\eta, X\left(t_{0}\right) \xi\right\rangle=\left\langle\eta, x_{0} \xi\right\rangle
\end{array}\right\}
$$

for arbitrary $\eta, \xi \in \mathbb{D} \underline{\otimes} \mathbb{E}$ and almost all $t \in I$.

Hence the existence of solution of (3.1) implies the existence of solution of (3.2) and vice-versa.

As explained in Ekhaguere [11], the sesquilinear form valued map P:

$$
P(t, x)(\eta, \xi) \neq \tilde{P}(t,\langle\eta, x \xi\rangle)
$$

For some complex-valued multifunction $\tilde{P}$ defined on $I \times C$ for $t \in I, x \in \tilde{\mathcal{A}}, \eta, \xi \in$ $D \otimes \mathbb{E}$.

The notion of solution of (3.1) or equivalently (3.2) is defined as follows:

Definition 3.2 : By a solution of (1.1) or equivalently (3.3), we mean a stochastic process $\varphi \in \operatorname{Ad}(\tilde{A})_{w a c} \cap L_{l o c}^{2}(\tilde{A})$ such that

$$
\begin{gathered}
d \varphi(x)=E(t, \varphi(t)) d \Lambda_{\pi}(t)+F(t, \varphi(t)) d A_{f}(t)+G(t, \varphi(t)) d A_{g}^{+}(t)+H(t, \varphi(t)) d t \\
\varphi\left(t_{0}\right) \quad=\varphi_{0} \text { almost all } t \in I .
\end{gathered}
$$

or equivalently

$$
\begin{array}{cc}
\frac{d}{d t}\langle\eta, \varphi(t) \xi\rangle & =P(t, \varphi(t))(\eta, \xi) \\
\left\langle\eta, \varphi\left(t_{0}\right) \xi\right\rangle & =\left\langle\eta, \varphi_{0} \xi\right\rangle
\end{array}
$$


for almost $\eta, \xi \in D \underline{\otimes \mathbb{E}}$.

\section{Definition 3.3}

(i) We denote the space of sesquilinear forms on $\mathbb{D} \underline{\otimes} \mathbb{E}$ by $\operatorname{sesq}(\mathbb{D} \underline{\otimes} \mathbb{E})$. Thus, $\operatorname{sesq}(\mathbb{D} \underline{\otimes} \mathbb{E})=\{a: \mathbb{D} \underline{\otimes} \mathbb{E} \times \mathbb{D} \underline{\otimes} \mathbb{E} \rightarrow \Phi \mid$ the map $(\eta, \xi) \rightarrow a(\eta, \xi)$ is linear in $\xi$ and conjugate linear in $\eta, \forall \eta, \xi \in \mathbb{D} \underline{\otimes} \mathbb{E}\}$.

(ii) Let $I \subseteq \mathbb{R}_{+}$, we denote by $L^{0}(I, \mathbb{D} \underline{\otimes} \mathbb{E})$ the set of all $\operatorname{ses} q(\mathbb{D} \underline{\otimes \mathbb{E}})$-valued maps on $I$, i.e.,

$$
L^{0}(I, \mathbb{D} \underline{\otimes} \mathbb{E})=\{u: I \rightarrow \operatorname{sesq}(\mathbb{D} \underline{\otimes} \mathbb{E})\} .
$$

Definition 3.4: We define $K(\eta, \xi)$ as follows:

Given any subset $K \in \operatorname{clos}(\tilde{\mathcal{A}})$, and any pair of elements $\eta, \xi \in \mathbb{D} \underline{\otimes \mathbb{E}}$,

$$
K(\eta, \xi)=\{<\eta, x \xi>: x \in K\} .
$$

Hence, $K(\eta, \xi) \subseteq \mathbb{C}$.

\section{VIABLE SOLUTIONS OF QUANTUM STOCHASTIC DIFFERENTIAL EQUATIONS}

Definition 4.1: Let $P: I \times \tilde{\mathcal{A}} \longrightarrow \operatorname{sesq}(\mathbb{D} \underline{\otimes} \mathbb{E})^{2}$ be a sesqulinear valued funtion, then the subset $K$ of $\tilde{\mathcal{A}}$ is viable with respect to $P$ if for every $\left(t_{0}, x_{0}\right) \in I \times K$ there exists $T \in I, T>t_{0}$ such that equation (3.3) have at least one solution $X \in K$ and that $<\eta, X \xi>\in K(\eta, \xi)$.

Definition 4.2 :For $K \in \operatorname{clos}(\tilde{\mathcal{A}}$, we assume that $K(\eta, \xi)$ is locally closed. That is, for each $x \in K,<\eta, x \xi>:=x_{\eta, \xi} \in K(\eta, \xi)$, there exists $\rho>0$ such that $D\left(x_{\eta, \xi}, \rho\right) \cap K(\eta, \xi)$ is closed for arbitrary pair $\eta, \xi \in \mathbb{D} \underline{\otimes} \mathbb{E}$.

Here, $D\left(x_{\eta, \xi}, \rho\right):=\left\{X_{\eta, \xi}:\left|x_{\eta, \xi}\right| \leq \rho\right\}$.

Next we define tangent cone as it applies to our present non commutative settings. We shall make use of the notion of the Bouligand- Severi tangency as in [17].

Definition 4.3: Let $K \subset \tilde{\mathcal{A}}, E \subset \tilde{\mathcal{A}}$ and $x \in K$. Then that $x_{\eta, \xi} \in K(\eta, \xi)$. The set $E(\eta, \xi)$ is said to be tangent to the set $K(\eta, \xi)$ at the point $x_{\eta \xi}$ if

$$
\liminf _{h \mapsto 0} \frac{1}{h} \mathbf{d}\left(x_{\eta, \xi}+h E(\eta, \xi) ; K(\eta, \xi)\right)=0 .
$$

We denote by $\mathcal{T}_{K(\eta, \xi)}$ the class of all sets which are tangent to $K(\eta, \xi)$ at the point $x_{\eta, \xi}$ for arbitrary $\eta, \xi \in D \underline{\otimes} \mathbb{E}$.

Proposition 4.1: The set $\mathcal{T}_{K(\eta, \xi)}\left(x_{\eta, \xi}\right)$ of all complex numbers which are tangent to the set $K(\eta, \xi)$ at the point $x_{\eta \xi}$ is a closed cone. 


\section{Proof}

Let $\left(x_{\eta \xi}\right) \in K(\eta, \xi)$;

by a preorder definition $E(\eta, \xi) \in \mathcal{T}_{K(\eta, \xi)}\left(x_{\eta \xi}\right)$ if

$$
\liminf _{t \mapsto 0} \frac{1}{t} \mathbf{d}\left(x_{\eta \xi}+t E(\eta, \xi) ; K(\eta, \xi)\right)=0
$$

Let $s>0$, then we have

$$
\begin{aligned}
\liminf _{t \mapsto 0} \frac{1}{t} \mathbf{d}\left(x_{\eta \xi}+t s E(\eta, \xi) ; K(\eta, \xi)\right) & =s \liminf _{t \mapsto 0} \frac{1}{t s} \mathbf{d}\left(x_{\eta \xi}+t s E(\eta, \xi) ; K(\eta, \xi)\right) \\
& =s \liminf _{\tau \mapsto 0} \frac{1}{\tau} \mathbf{d}\left(x_{\eta \xi}+\tau E(\eta, \xi) ; K(\eta, \xi)\right)
\end{aligned}
$$

Hence, $s E(\eta, \xi) \in \mathcal{T}_{K}\left(x_{\eta \xi}\right)$

To complete the proof, we need to show that $\mathcal{T}_{K}\left(x_{\eta \xi}\right)$ is a closed set.

Let $\mathbb{N}^{*}$ be the set of strictly positive natural numbers.

Let $\left(E_{n}(\eta, \xi)\right)_{n \in \mathbb{N}^{*}}$ be a sequence of elements in $\mathcal{T}_{K}\left(x_{\eta \xi}\right)$, convergent to $E(\eta, \xi)$ then we have

$$
\begin{aligned}
\frac{1}{t} \mathbf{d}\left(x_{\eta \xi}+t E(\eta, \xi) ; K(\eta, \xi)\right) & \leq \frac{1}{t}\left|t\left(E(\eta, \xi)-E_{n}(\eta, \xi)\right)\right|+\frac{1}{t} \mathbf{d}\left(x_{\eta \xi}+t E_{n}(\eta, \xi) ; K(\eta, \xi)\right) \\
& =\left|E(\eta, \xi)-E_{n}(\eta, \xi)\right|+\frac{1}{t} \mathbf{d}\left(x_{\eta \xi}+t E_{n}(\eta, \xi) ; K(\eta, \xi)\right)
\end{aligned}
$$

for every $n \in \mathbb{N}^{*}$. So

$$
\liminf _{t \mapsto 0} \frac{1}{t} \mathbf{d}\left(x_{\eta \xi}+t E(\eta, \xi) ; K(\eta, \xi)\right) \leq\left|E(\eta, \xi)-E_{n}(\eta, \xi)\right|
$$

for every $n \in \mathbb{N}^{*}$.

Since $\lim _{n \rightarrow \infty}\left|E(\eta, \xi)-E_{n}(\eta, \xi)\right|=0$, then

$$
\liminf _{t \mapsto 0} \frac{1}{t} \mathbf{d}\left(x_{\eta \xi}+t E(\eta, \xi) ; K(\eta, \xi)\right)=0
$$

which shows that the set $\mathcal{T}_{K(\eta, \xi)}\left(x_{\eta \xi}\right)$ is a closed cone.

Proposition 4.2: A set $E(\eta, \xi) \in \mathbb{C}$ belongs to the cone $\mathcal{T}_{K(\eta, \xi)}\left(x_{\eta, \xi}\right)$ if and only if for every $\epsilon>0$ there exists $h \in(0, \epsilon)$ and $q_{\eta \xi, h} \in D_{\eta, \xi}(0, \epsilon)$ with the property

$$
x_{\eta \xi}+h\left(E(\eta, \xi)+q_{\eta \xi, h}\right) \in K(\eta, \xi) .
$$

\section{Proof}

From the proof of proposition 4.1 we see that $E(\eta, \xi) \in \mathcal{T}_{K(\eta, \xi)}\left(x_{\eta \xi}\right)$ if and only 
if

for every $\epsilon>0$ there exists $h \in(0, \epsilon)$ and $p_{\eta \xi, h} \in K(\eta, \xi)$ such that

$$
\frac{1}{h}\left|x_{\eta \xi}+h E(\eta, \xi)-p_{\eta \xi, h}\right| \leq \epsilon
$$

Now, let us define

$$
q_{\eta \xi, h}=\frac{1}{h}\left(p_{\eta \xi, h}-x_{\eta \xi}-h E(\eta, \xi)\right),
$$

and we observe that, we have both $\left|q_{\eta \xi, h}\right| \leq \epsilon$ and

$$
x_{\eta \xi}+h\left(E(\eta, \xi)+q_{\eta \xi, h}\right)=p_{\eta \xi, h} \in K(\eta, \xi) .
$$

Definition 4.4: Let $K \in \operatorname{clos}(\tilde{\mathcal{A}})$ and $I \subseteq \mathbb{R}_{+}$.

(i) A map $\Phi: I \times K \longrightarrow \operatorname{clos}(\tilde{\mathcal{A}})$ will be called Lipschitzian if for each $\eta, \xi \in \mathbb{D} \underline{\otimes \mathbb{E}}$, there exists $k_{\eta, \xi}^{\Phi}: I \rightarrow(0, \infty)$ in $L_{l o c}^{1}(I)$ such that

$$
\rho_{\eta, \xi}(\Phi(t, x), \Phi(t, y)) \leq k_{\eta, \xi}^{\Phi}(t)\|x-y\|_{\eta, \xi}
$$

for all $x, y \in K$ and almost all $t \in I$

(ii) If $\Phi$ is a sesqulinear form valued multifunction then the map $\Phi: I \times K \longrightarrow$ $2^{\operatorname{sesq}(\mathbb{D} \underline{\otimes})}$ will be said to be Lipschitzian (resp. continuous) if for arbitrary $\eta, \xi \in \mathbb{D} \otimes \mathbb{E}$, the map $(t, x) \rightarrow \Phi(t, x)(\eta, \xi)$ from $I \times K$ to $\mathscr{Q}^{\mathbb{Q}}$ is Lipschitzian (resp. continuous).

(iii) If $\Phi: I \times K \longrightarrow \operatorname{sesq}(\mathbb{D} \underline{\otimes \mathbb{E}})$ will be said to be Lipschitzian if for arbitrary $\eta, \xi \in \mathbb{D} \underline{\otimes} \mathbb{E}$, the map

$$
\mid\left(\Phi(t, x)(\eta, \xi)-\Phi(t, y)(\eta, \xi) \mid \leq k_{\eta, \xi}^{\Phi}(t)\|x-y\|_{\eta, \xi}\right.
$$

Definition 4.5: A map $P: I \times K \longrightarrow \operatorname{sesq}(\mathbb{D} \underline{\otimes \mathbb{E}})$ is said to be Lipschitzian and quasi-compact if the following conditions are satisfied :

$\left(Q_{1}\right)$ there exists a continuous map $\mathcal{P}: I \times K^{2} \rightarrow \operatorname{sesq}(\mathbb{D} \underline{\otimes \mathbb{E}})$ such that for each $(t, x) \in I \times K$

$$
P(t, x)(\eta, \xi)=\mathcal{P}(t, x, x)(\eta, \xi),
$$

and for each $t_{0} \in\left[t_{0}, T\right]$ and $x_{0} \in K$, there exists $\rho>0$ such that $D\left(x_{0}, \rho\right)(\eta, \xi) \cap$ $K(\eta, \xi)$ is closed.

$\left(Q_{2}\right)$ each sequence $\left\{x_{n}\right\}_{n}$, from $D\left(x_{0}, \rho\right) \cap K$ has at least one subsequence $\left\{x_{n_{k}}\right\}_{k}$ such that $\left(\mathcal{P}\left(t, x_{n_{k}}, y\right)(\eta, \xi)\right)$ is Cauchy for $(t, y) \in\left[t_{0}, T\right] \times\left(D\left(x_{0}, \rho\right) \cap K\right)$.

That it is, for each $\epsilon>0$, there exists $k(\epsilon)>0$ such that for each $k, p \geq k(\epsilon)$ we have

$$
\left|\mathcal{P}\left(t, x_{n_{k}}, y\right)(\eta, \xi)-\mathcal{P}\left(t, x_{n_{p}}, y\right)(\eta, \xi)\right| \leq \epsilon
$$


$\left(Q_{3}\right)$ there exists $K_{\eta, \xi}^{\mathcal{P}}: I \rightarrow(0, \infty)$, in $L_{l o c}^{1}(I)$ such that

$$
|\Phi(t, x, y)(\eta, \xi)-\Phi(t, x, w)(\eta, \xi)| \leq k_{\eta \xi}^{\mathcal{P}}\|y-w\|_{\eta \xi}
$$

for each $t \in\left[t_{0}, T\right]$ and $x, y, w \in D\left(x_{0}, \rho\right) \cap K$.

$\left(Q_{4}\right)$ the $\operatorname{map}\left\{x \longrightarrow \mathcal{P}(t, x, y)(\eta, \xi):(t, y) \in\left[t_{0}, T\right] \times D\left(x_{0}, \rho\right) \cap K\right\}$ is uniformly equicontinuous on $D\left(x_{0}, \rho\right) \cap K$ i.e given $\epsilon>0$, there exists $\delta(\epsilon)>0$ such that

$$
|\mathcal{P}(t, x, y)(\eta, \xi)-\mathcal{P}(t, \bar{x}, y)(\eta, \xi)| \leq \epsilon
$$

for each $t \in\left[t_{0}, T\right]$ and $x, \bar{x}, y \in D\left(x_{0}, \rho\right) \cap K(\eta, \xi)$ with $\|x-\bar{x}\|_{\eta \xi} \leq \delta(\epsilon)$.

\section{MAIN RESULTS ON THE EXISTENCE OF VIABLE SOLUTIONS}

This section concerns the establishment of the existence of viable solutions of quantum stochastic differential equation in which the coefficients are Lipschitzian and quasi compact. In so doing, a number of auxilliary results will be needed. The following results will be used to establish the main results of this paper.

\section{Theorem 5.1[Brezis - Browder ordering principle] [17]}

Let $\mathcal{S}$ be a non empty set, $\preceq$ a preorder on $\mathcal{S}$ and let $\mathcal{M}: \mathcal{S} \longrightarrow \mathbb{R} \cup\{+\infty\}$ be a function. Suppose that :

(i) for any increasing sequence $\left(\zeta_{k}\right)_{k}$ in $\mathcal{S}$, there exists some $\eta \in \mathcal{S}$ such that $\zeta_{k} \preceq \eta$ for all $k \in \mathbb{N}$;

(ii) the function $\mathcal{M}$ is increasing.

Then for each $\zeta_{0} \in \mathcal{S}$ there exists an $\mathcal{M}$-maximal element $\bar{\zeta} \in \mathcal{S}$ satisfying $\zeta_{0} \preceq \bar{\zeta}$.

Theorem 5.2. Suppose $K \subset \tilde{\mathcal{A}}$, satisfying the following

(a) $K \in \operatorname{clos}(\tilde{\mathcal{A}}), K \neq \emptyset$.

(b) $P\left(t_{0}, x_{0}\right)(\eta, \xi) \in \mathcal{T}_{\mathcal{K}(\eta, \xi)}\left(x_{0, \eta \xi}\right)$ for each pair $\left(t_{0}, x_{0}\right) \in I \times K$.

Then, for each $\epsilon \in(0,1)$, there exists a non decreasing function $\sigma:\left[t_{0}, T\right] \rightarrow\left[t_{0}, T\right]$ and two stochastic processes $g:\left[t_{0}, T\right] \rightarrow \tilde{\mathcal{A}}$ and $\varphi:\left[t_{0}, T\right] \rightarrow \tilde{\mathcal{A}}$ lying in $\operatorname{Ad}(\tilde{\mathcal{A}})_{\text {wac }} \cap L_{\text {loc }}^{2}(\tilde{\mathcal{A}})$ such that the corresponding sesquilinear form valued maps associated with any pair of $\eta, \xi \in \mathbb{D} \underline{\otimes \mathbb{E}})$ defined by $g:\left[t_{0}, T\right] \rightarrow \operatorname{ses} q(\mathbb{D} \otimes \mathbb{E})^{2}$ where $g(t)(\eta, \xi)=\langle\eta, g(t) \xi\rangle$ and $\varphi:\left[t_{0}, T\right] \rightarrow \operatorname{ses}(\mathbb{D} \underline{\otimes} \mathbb{E})^{2}$ where $\varphi(t)(\eta, \xi)=\langle\eta, \varphi(t) \xi\rangle$ satisfy the following for every $t \in\left[t_{0}, T\right]$,

(i) $t-\epsilon \leq \sigma(t) \leq t,\left|g_{\eta \xi}(t)\right| \leq \epsilon$,

(ii) $\varphi_{\eta \xi}(\sigma(t)) \in D\left(x_{0}, \rho\right)(\eta, \xi) \cap K(\eta, \xi)$ and $\varphi_{\eta \xi}(T) \in D\left(x_{0}, \rho\right)(\eta, \xi) \cap K(\eta, \xi)$ 
(iii) $\varphi$ satisfies

$$
\langle\eta, \varphi(t) \xi\rangle=\left\langle\eta, x_{0} \xi\right\rangle+\int_{t_{0}}^{t} P\left(\sigma(s), \varphi(\sigma(s))(\eta, \xi) d s+\int_{t_{0}}^{t} g(s)(\eta, \xi) d s\right.
$$

for every $t \in\left[t_{0}, T\right]$.

Remark. A pair of the triple $(\sigma, g, \varphi)$ with the associated complex valued functions $\left(\sigma, g_{\eta \xi}, \varphi_{\eta \xi}\right)$ satisfying (i), (ii), (iii) will be called an $\epsilon$ - approximate solution to the problem (3.3) on the interval $\left[t_{0}, T\right]$

\section{Proof of the Existence of Approximate Solutions :}

Let $\left(t_{0}, x_{0}\right) \in I \times K$ then $\left(t_{0}, x_{0, \eta \xi}\right) \in I \times K(\eta, \xi)$. Let $\rho>0$, then $D\left(x_{0}, \rho\right)(\eta, \xi) \cap$ $K(\eta, \xi)$ is closed in the set of complex numbers. There exists a constant $M_{\eta \xi}>0$, such that

$$
|P(t, x)(\eta, \xi)| \leq M_{\eta \xi}
$$

for every $t \in\left[t_{0}, T\right]$ and $x \in D(x, \rho) \cap K$ and

$$
<\eta, x \xi>:=x_{\eta \xi} \in D\left(x_{0}, \rho\right)(\eta, \xi) \cap K(\eta, \xi)
$$

where

$$
\left(T-t_{0}\right)\left(M_{\eta \xi}+1\right) \leq \rho
$$

Let $t_{0} \in I, \quad x_{0, \eta \xi} \in K(\eta, \xi)$ and let $\rho>0, M_{\eta \xi}>0$ be as above. Let $\epsilon \in(0,1)$.

We first establish the existence of an $\epsilon$ - approximate solution on an interval $\left[t_{0}, c\right]$ with $c \in\left(t_{0}, T\right]$. Since for every

$$
\left(t_{0}, x_{0, \eta \xi}\right) \in I \times K(\eta, \xi), \quad P\left(t_{0}, x_{0}\right)(\eta, \xi) \in \mathcal{T}_{\mathcal{K}(\eta, \xi)}\left(x_{0, \eta \xi}\right)
$$

from Proposition (2), it follows that there exists

$$
c \in\left(t_{0}, T\right], c-t_{0} \leq \epsilon, \text { and } q_{\eta \xi, h} \in \mathbb{C} \text { with }\left|q_{\eta \xi, h}\right| \leq \epsilon
$$

such that

$$
x_{0, \eta \xi}+\left(c-t_{0}\right) P\left(t_{0}, x_{0}\right)(\eta, \xi)+\left(c-t_{0}\right) q_{\eta \xi, h} \in K(\eta, \xi) .
$$


Let $I_{c}=\left[t_{0}, c\right]$, we now define the function $\sigma:\left[t_{0}, c\right] \longrightarrow\left[t_{0}, c\right]$, and stochastic processes $g:\left[t_{0}, c\right] \longrightarrow \tilde{\mathcal{A}}$ and $\varphi:\left[t_{0}, c\right] \longrightarrow \tilde{\mathcal{A}}$ lying in $A d(\tilde{\mathcal{A}})_{w a c} \cap L_{l o c}^{2}(\tilde{\mathcal{A}})$ satisfying

$$
\begin{cases}\sigma(t)=t_{0} & \text { for } t \in\left[t_{0}, c\right],, \\ g_{\eta \xi}(t)=q_{\eta \xi} & \text { for } t \in\left[t_{0}, c\right], \\ \varphi_{\eta \xi}(t)=x_{0, \eta \xi}+\left(t-t_{0}\right) P\left(t_{0}, x_{0}\right)(\eta, \xi)+\left(t-t_{0}\right) q_{\eta \xi}, & \text { for } t \in\left[t_{0}, c\right] .\end{cases}
$$

The triple $\left(\sigma, g_{\eta \xi}, \varphi_{\eta \xi}\right)$ is an $\epsilon$ - approximate solution to problem (3.3) on the interval $\left[t_{0}, c\right]$. This shows that conditions (i), (ii) and (iv) are satisfied, we now show that condition (iii) is also satisfied by uniqueness of solutions, using (5.2),(5.3) and (i). From (i) $\sigma(t)=t_{0}$ and $\left\langle\eta, X\left(t_{0}\right) \xi\right\rangle=\left\langle\eta, x_{0} \xi\right\rangle$, by equation (3.3)then

$$
\langle\eta, \varphi(\sigma(t)) \xi\rangle=\left\langle\eta, x_{0} \xi\right\rangle
$$

Therefore we have

$$
\varphi(\sigma(t))(\eta, \xi) \in D\left(x_{0, \eta \xi}, \rho\right) \cap K(\eta, \xi) \forall t \in\left[t_{0}, c\right]
$$

Therefore,

$$
\varphi(c)(\eta, \xi) \in K(\eta, \xi)
$$

Moreover, by (5.2) and (5.3),we have

$\left|\varphi(c)(\eta, \xi)-\varphi_{0}(\eta, \xi)\right| \leq\left(c-t_{0}\right)\left|P\left(t_{0}, \varphi_{0}\right)(\eta, \xi)\right|+\left(c-t_{0}\right)\left|q_{\eta \xi}\right| \leq\left(T-t_{0}\right)\left(M_{\eta \xi}+1\right) \leq \rho$.

For every $t \in\left[t_{0}, c\right]$. Thus (iii) is also satisfied.

We now define the $\epsilon$ - approximate solution on the whole interval $I$. We make use of Brezis-Browder Theorem

Let $\mathcal{S}$ be the set of all $\epsilon$ - approximate solutions to the problem (4.3) defined on the interval $\left[t_{0}, c\right]$ with $c \in\left(t_{0}, T\right]$.

On $\mathcal{S}$ we define the relation “ $\preceq^{\prime \prime}$ by $\left(\sigma_{1}, g_{1, \eta \xi}, \varphi_{1, \eta \xi}\right) \preceq\left(\sigma_{2}, g_{2, \eta \xi}, \varphi_{2, \eta \xi}\right)$ if the domain of definition $\left[t_{0}, c_{1}\right]$ of the first triple is included in the domain of definition $\left[t_{0}, c_{2}\right]$ of the second triple, and the two $\epsilon$ - approximate solutions coincide on the common part of the domains. Then, "preceq" is a pre-order relation on $\mathcal{S}$. 
Firstly, we show that each increasing sequence $\left(\left(\sigma_{m}, g_{m, \eta \xi}, \varphi_{m, \eta \xi}\right)\right)_{m}$ is bounded from above .

Let $\left(\left(\sigma_{m}, g_{m, \eta \xi}, \varphi_{m, \eta \xi}\right)\right)_{m}$ be an increasing sequence, and let

$$
c^{*}=\lim _{m} c_{m}
$$

where $\left[t_{0}, c_{m}\right]$ denotes the domain of definition of $\left(\sigma_{m}, g_{m, \eta \xi}, \varphi_{m, \eta \xi}\right)$. Then $c^{*} \in\left(t_{0}, T\right]$.

We will show that there exists at least one element, $\left(\sigma^{*}, g_{\eta \xi}^{*}, \varphi_{\eta \xi}^{*}\right) \in \mathcal{S}$, defined on $\left[t_{0}, c^{*}\right]$ and satisfying

$$
\left(\sigma_{m}, g_{m, \eta \xi}, \varphi_{m, \eta \xi}\right) \preceq\left(\sigma^{*}, g_{\eta \xi}^{*}, x_{\eta \xi}^{*}\right)
$$

for each $m \in \mathbb{N}$. In order to do this, we first prove that there exists

$$
\lim _{m} \varphi_{m}\left(c_{m}\right)(\eta, \xi) \text {. }
$$

For each $m, n \in \mathbb{N}, m \leq n$ we have $\varphi_{m}(s)=\varphi_{n}(s)$ for all $s \in\left[t_{0}, c_{m}\right]$. Taking into account (iii), (iv) and (5.2), we have

$$
\begin{aligned}
& \left|\varphi_{m}\left(c_{m}\right)(\eta, \xi)-\varphi_{n}\left(c_{n}\right)(\eta, \xi)\right| \\
\leq & \int_{c_{m}}^{c_{n}}\left|P\left(\sigma_{n}(s), \varphi_{n}\left(\sigma_{n}(s)\right)\right)(\eta, \xi)\right| d s+\int_{c_{m}}^{c_{n}}\left|g_{n}(s)(\eta, \xi)\right| d s \\
\leq & \left(M_{\eta \xi}+\epsilon\right)\left|c_{n}-c_{m}\right|
\end{aligned}
$$

for every $m, n \in \mathbb{N}$, which shows that there exists

$$
\lim _{m \mapsto \infty} \varphi_{m}\left(c_{m}\right)(\eta, \xi) \in D\left(x_{0}, \rho\right)(\eta, \xi) \cap K(\eta, \xi) .
$$

Furthermore, because all the functions in the set $\left\{\sigma_{m}: m \in \mathbb{N}\right\}$ are non decreasing, with values in $\left[t_{0}, c^{*}\right]$, and satisfy $\sigma_{m}\left(c_{m}\right) \leq \sigma_{p}\left(c_{p}\right)$ for every $m, p \in \mathbb{N}$, there exists $\lim _{m \rightarrow \infty} \sigma_{m}\left(c_{m}\right)$ and belongs to $\left[t_{0}, c^{*}\right]$. We now define a triple function $\left(\sigma^{*}, g_{\eta \xi}^{*}, \varphi_{\eta \xi}^{*}\right):\left[t_{0}, c^{*}\right] \longrightarrow\left[t_{0}, c^{*}\right] \times \mathbb{C} \times \mathbb{C}$ by

$$
\begin{aligned}
& \sigma^{*}(t)= \begin{cases}\sigma_{m}(t) & \text { for } t \in\left[t_{0}, c_{m}\right], m \in \mathbb{N}, \\
\lim _{m \mapsto \infty} \sigma_{m}\left(c_{m}\right) & \text { for } t=c^{*},\end{cases} \\
& g_{\eta \xi}^{*}(t)= \begin{cases}g_{m}(t)(\eta, \xi) & \text { for } t \in\left[t_{0}, c_{m}\right], m \in \mathbb{N}, \text { for any } \eta, \xi \in \mathbb{D} \underline{\otimes \mathbb{E}),} \\
0 & \text { for } t=c^{*},\end{cases} \\
& \varphi_{\eta \xi}^{*}(t)= \begin{cases}\varphi_{m}(t)(\eta, \xi) & \text { for } \left.t \in\left[t_{0}, c_{m}\right], m \in \mathbb{N}, \text { for any } \eta, \xi \in \mathbb{D} \underline{\otimes \mathbb{E}}\right), \\
\lim _{m \mapsto \infty} \varphi_{m}\left(c_{m}\right)(\eta, \xi) & \text { for } t=c^{*},\end{cases}
\end{aligned}
$$


This shows that $\left(\sigma^{*}, g_{\eta \xi}^{*}, \varphi_{\eta \xi}^{*}\right)$ is an $\epsilon$ - approximate solution which is an upper bound for $\left(\left(\sigma_{m}, g_{m, \eta \xi}, \varphi_{m, \eta \xi}\right)\right)_{m}$. Applying (ii) of Brezis-Browder theorem(5.1), we define the function

$\mathcal{M}: \mathcal{S} \longrightarrow \mathbb{R} \cup\{+\infty\}$. Then, for each $\zeta_{0} \in \mathcal{S}$ there exists an $\mathcal{M}$ - maximal element $\bar{\zeta} \in \mathcal{S}$ satisfying $\zeta_{0} \preceq \bar{\zeta}$. This shows that

$$
\mathcal{M}\left(\left(\sigma, g_{\eta \xi}, \varphi_{\eta \xi}\right)\right)=c
$$

, where $\left[t_{0}, c\right]$ is the domain of definition of $\left(\sigma, g_{\eta \xi}, \varphi_{\eta \xi}\right)$. This shows that $\mathcal{M}$ satisfies the hypothesis of Brezis- Browder theorem (5.1).

Then $\mathcal{S}$ contains at least one $\mathcal{M}$ - maximal element $\left(\bar{\sigma}, \bar{g}_{\eta \xi}, \bar{\varphi}_{\eta \xi}\right)$ defined on $\left[t_{0}, \bar{c}\right]$. If $\left(\tilde{\sigma}, \tilde{g}_{\eta \xi}, \tilde{\varphi}_{\eta \xi}\right) \in \mathcal{S}$, defined on $\left[t_{0}, \tilde{c}\right]$, satisfies $\left(\bar{\sigma}, \bar{g}_{\eta \xi}, \bar{\varphi}_{\eta \xi}\right) \preceq\left(\tilde{\sigma}, \tilde{g}_{\eta \xi}, \tilde{\varphi}_{\eta \xi}\right)$, then we necessarily have $\bar{c}=\tilde{c}$. We will show next that $\bar{c}=T$. we assume by contradiction that $\bar{c}<T$. Then, taking into account the fact that $\bar{\varphi}_{\eta \xi}(\bar{c}) \in D\left(x_{0}, \rho\right)(\eta, \xi) \cap K(\eta, \xi)$, we have

$$
\begin{aligned}
& \left|\bar{\varphi}_{\eta \xi}(\bar{c})-x_{0, \eta \xi}\right| \\
\leq & \int_{t_{0}}^{\bar{c}}|P(\bar{\sigma}(s), \bar{\varphi}(\bar{\sigma}(s)))(\eta, \xi)| d s+\int_{t_{0}}^{\bar{c}}|\bar{g}(\eta, \xi)(s)| d s \\
\leq & \left(\bar{c}-t_{0}\right)\left(M_{\eta \xi}+\epsilon\right) \\
\leq & \left(\bar{c}-t_{0}\right)\left(M_{\eta \xi}+1\right)<\left(T-t_{0}\right)\left(M_{\eta \xi}+1\right) \leq \rho
\end{aligned}
$$

Then, as $\bar{\varphi}_{\eta \xi}(\bar{c}) \in K(\eta, \xi)$ and $P(\bar{c}, \bar{\varphi}(\bar{c}))(\eta, \xi) \in \mathcal{T}_{K}(\bar{\varphi}(\bar{c}))(\eta, \xi)$, there exists $\delta(0, T-\bar{c}), \delta \leq \epsilon$ and $q_{\eta \xi} \in \mathbb{C}$ such that $\left|q_{\eta \xi}\right| \leq \epsilon$ and

$$
\overline{\varphi_{\eta \xi}}(\bar{c})+\delta P(\bar{c}, \bar{\varphi}(\bar{c}))(\eta, \xi)+\delta q_{\eta \xi} \in K(\eta, \xi)
$$

From the inequality above we have

$$
\left.\mid \bar{\varphi}(\bar{c})(\eta, \xi)+\delta P(\bar{c}, \bar{\varphi}(\bar{c}))(\eta, \xi)+q_{\eta \xi}\right]-\varphi_{0}(\eta, \xi) \mid \leq \rho
$$

We now define the functions

$\sigma:\left[t_{0}, \bar{c}+\delta\right] \longrightarrow\left[t_{0}, \bar{c}+\delta\right]$ and $g:\left[t_{0}, \bar{c}+\delta\right] \longrightarrow \mathbb{C}$ by

$$
\begin{gathered}
\sigma(t)= \begin{cases}\bar{\sigma}(t) & \text { for } t \in\left[t_{0}, \bar{c}\right], \\
\bar{c} & \text { for } t \in[\bar{c}, \bar{c}+\delta],\end{cases} \\
g_{\eta \xi}(t)= \begin{cases}\bar{g}_{\eta \xi}(t) & \text { for } \left.t \in\left[t_{0}, \bar{c}\right], \text { and for any } \eta, \xi \in \mathbb{D} \underline{\otimes} \mathbb{E}\right), \\
q & \text { for } t \in[\bar{c}, \bar{c}+\delta],\end{cases}
\end{gathered}
$$


so $\left|g_{\eta \xi}(t)\right| \leq \epsilon$ for every $t \in\left[t_{0}, \bar{c}+\delta\right]$. In addition, for every $t \in\left[t_{0}, \bar{c}+\delta\right], \sigma(t) \in\left[t_{0}, \bar{c}\right]$ and therefore $\bar{\varphi}(\sigma(t))$ is well-defined and belongs to the set $D\left(x_{0}, \rho\right)(\eta, \xi) \cap K(\eta, \xi)$. Accordingly, we can define $\varphi_{\eta \xi}:\left[t_{0}, \bar{c}+\delta\right] \longrightarrow \mathbb{C}$ by

$$
\langle\eta, \varphi(t) \xi\rangle=\left\langle\eta, \varphi_{0} \xi\right\rangle+\int_{t_{0}}^{t} P(\sigma(s), \bar{\varphi}(\sigma(s)))(\eta, \xi) d s+\int_{t_{0}}^{t} g(\eta, \xi)(s) d s
$$

for every $t \in\left[t_{0}, \bar{c}+\delta\right]$. clearly, $\varphi_{\eta, \xi}$ coincides with $\varphi_{\eta, \xi}^{-}$on $\left[t_{0}, \bar{c}\right]$ since the domain $\left[t_{0}, \bar{c}\right]$ is included in the domain of $[\bar{c}, \bar{c}+\delta]$ and then it readily follows that $\varphi_{\eta, \xi}, \sigma$ and $g_{\eta, \xi}$ satisfy all the conditions in (i) and (ii). In order to prove (iii) and (iv) we observe that

$$
\varphi_{\eta \xi}(t) \in \begin{cases}\bar{\varphi}_{\eta \xi}(t) & \text { for } t \in\left[t_{0}, \bar{c}\right] . \\ \varphi_{\eta \xi}(\bar{c})+(t-\bar{c}) P(\bar{c}, \bar{\varphi}(\bar{c}))(\eta, \xi)+(t-\bar{c}) q & \text { for } t \in[\bar{c}, \bar{c}+\delta]\end{cases}
$$

Then $\varphi_{\eta \xi}$ satisfies the equation in (iv). since

$$
\varphi_{\eta \xi}(\sigma(t)) \in \begin{cases}\bar{\varphi}_{\eta \xi} \bar{\sigma}(t) & \text { for } t \in\left[t_{0}, \bar{c}\right] \\ \bar{\varphi}_{\eta \xi}(\bar{c}) & \text { for } t \in\left[t_{0}, \bar{c}+\delta\right]\end{cases}
$$

it follows that $\varphi_{\eta \xi}(\sigma(t)) \in D\left(x_{0}, \rho\right)(\eta, \xi) \cap K(\eta, \xi)$. Furthermore, from the choice of $\delta$ and $q_{\eta \xi}$, we have both

$$
\begin{aligned}
\varphi_{\eta \xi}(\bar{c}+\delta) \in \overline{\varphi_{\eta \xi}}(\bar{c}) & (\eta, \xi)+\delta \mathbb{P}(\bar{c}, \bar{\varphi}(\bar{c}))(\eta, \xi)+\delta q \in K(\eta, \xi) \text { and } \\
& \left|\varphi(\bar{c}+\delta)(\eta, \xi)-x_{0}(\eta, \xi)\right| \\
\leq & \left|\bar{\varphi}(\bar{c})(\eta, \xi)+\delta P(\bar{c}, \bar{\varphi}(\bar{c}))(\eta, \xi)+\delta q-x_{0}(\eta, \xi)\right| \\
\leq & \rho
\end{aligned}
$$

and consequently, $\varphi_{\eta \xi}$ satisfies (iii). Thus $\left(\sigma, g_{\eta \xi}, \varphi_{\eta \xi}\right) \in \mathcal{S}$.

Furthermore, since $\left(\bar{\sigma}, \bar{g}_{\eta \xi}, \bar{\varphi}_{\eta \xi}\right) \preceq\left(\sigma, g_{\eta \xi}, \varphi_{\eta \xi}\right)$ and $\bar{c}<\bar{c}+\delta$, it follows that $\left(\bar{\sigma}, \bar{g}_{\eta \xi}, \bar{\varphi}_{\eta \xi}\right)$ is not a $\mathcal{M}$ - maximal element. But this is absurd, we can eliminate this contradiction, only if each maximal element in the set $\mathcal{S}$ is defined on $\left[t_{0}, T\right]$. Hence $\bar{c}=T$

We now establish the main theorem of this paper.

\section{Theorem 5.3}

Let $K \subset \tilde{\mathcal{A}}, K \neq \emptyset$ and $K(\eta, \xi)=\{\langle\eta, x \xi\rangle: x \in K, \eta, \xi \in \mathbb{D} \underline{\otimes \mathbb{E}})\}$

Assume that the following conditions hold:

(i) The map $(t, x) \longrightarrow P(t, x)(\eta, \xi)$ associated with the right hand-side of (3.3) is Lipschitzian and quasi-compact.

(ii) There exists $M_{\eta \xi}>0$ such that $|P(t, x)(\eta, \xi)| \leq M_{\eta \xi}$ for every $t \in\left[t_{0}, T\right]$ and $x \in D\left(x_{0}, \rho\right) \cap K$ 
(iii) $\left(T-t_{0}\right)\left(M_{\eta \xi}+1\right) \leq \rho$

Then $K(\eta, \xi)$ is viable with respect to $P$ if and only if for every $\left(t_{0}, x_{0}\right) \in I \times K$ we have $P\left(t_{0}, x_{0}\right)(\eta, \xi) \in \mathcal{T}_{K(\eta, \xi)}\left(x_{0, \eta \xi}\right)$

\section{Proof}

Suppose $K$ is viable with respect to $P$, then there exists a solution $\varphi$ that satisfy equation (3.3).

Let $\left(t_{0}, x_{0}\right) \in I \times K$, then $\left(t_{0}, x_{0, \eta \xi}\right) \in I \times K(\eta, \xi)$.

We prove that

$$
\lim _{h \mapsto 0} \frac{1}{h} \mathbf{d}\left(x_{0, \eta \xi}+h P\left(t_{0}, x_{0}\right)(\eta, \xi) ; K(\eta, \xi)\right)=0
$$

and a stochastic process $\varphi \in K$ with $\left\langle\eta, \varphi\left(t_{0}\right) \xi\right\rangle \in K(\eta, \xi)$ satisfying equation (3.3).

Let

$$
\varphi_{\eta \xi}\left(t_{0}+h\right)=\left\langle\eta, \varphi\left(t_{0}+h\right) \xi\right\rangle
$$

and

$$
\varphi_{\eta \xi}\left(t_{0}\right)=\left\langle\eta, \varphi\left(t_{0}\right) \xi\right\rangle
$$

then

$$
\begin{gathered}
\varphi_{\eta \xi}\left(t_{0}+h\right)-\varphi_{\eta \xi}\left(t_{0}\right)=\left\langle\eta, \varphi\left(t_{0}+h\right) \xi\right\rangle-\left\langle\eta, \varphi\left(t_{0}\right) \xi\right\rangle \\
=\left\langle\eta,\left[\varphi\left(t_{0}+h\right)-\varphi\left(t_{0}\right)\right] \xi\right\rangle \\
\quad \mathbf{d}\left(x_{0, \eta \xi}+h P\left(t_{0}, x_{0}\right)(\eta, \xi) ; K(\eta, \xi)\right) \\
\leq\left|x_{0, \eta \xi}+h P\left(t_{0}, x_{0}\right)(\eta, \xi)-\left\langle\eta, \varphi\left(t_{0}\right) \xi\right\rangle\right| \\
=\lim _{h \mapsto 0} \frac{1}{h}\left|x_{0, \eta \xi}+h P\left(t_{0}, x_{0}\right)(\eta, \xi)-\left\langle\eta, \varphi\left(t_{0}+h\right) \xi\right\rangle\right| \\
=\lim _{h \mapsto 0}\left|P\left(t_{0}, \varphi\left(t_{0}\right)\right)(\eta, \xi)-\frac{\left\langle\eta,\left(\varphi\left(t_{0}+h\right)-\varphi\left(t_{0}\right)\right) \xi\right\rangle}{h}\right| \\
=\left|P\left(t_{0}, \varphi\left(t_{0}\right)\right)(\eta, \xi)-\lim _{h \mapsto 0} \frac{\left\langle\eta,\left(\varphi\left(t_{0}+h\right)-\varphi\left(t_{0}\right)\right) \xi\right\rangle}{h}\right| \\
=\left|P\left(t_{0}, \varphi\left(t_{0}\right)\right)(\eta, \xi)-\frac{d}{d t}\langle\eta, \varphi(t) \xi\rangle\right|_{t=t_{0}} \\
=0
\end{gathered}
$$

This shows that the stochastic process $\varphi$ is a solution to equation (3.3) and belongs to $K$.

Suppose $P\left(t_{0}, x_{0}\right)(\eta, \xi) \in \mathcal{T}_{\mathcal{K}(\eta, \xi)}$ then we prove that $P$ is viable to $K$ This concerns the existence and convergence of approximate solutions. 
Theorem 5.2 above has established the existence of approximate solution.

Next, we prove the convergence of a suitably chosen sequence of approximate solutions.

Let $\left\{\epsilon_{n}\right\}_{n \in \mathbb{N}}$ be a sequence in $(0,1)$ decreasing to 0 and let $\left(\sigma_{n}, g_{n, \eta \xi}, \varphi_{n, \eta \xi}\right)_{n \in \mathbb{N}}$ be a sequence of $\epsilon_{n}$-approximate solutions of (3.3) defined on $\left[t_{0}, T\right]$.

We will show first that $\left(\varphi_{n, \eta \xi}\left(\sigma_{n}\right)\right)_{n \in \mathbb{N}}$ has at least one Cauchy subsequence.

For $n, m \in N$ and $s \in\left[t_{0}, T\right]$, let

$$
p_{n, m}(s)(\eta, \xi)=\mathcal{P}\left(s, \varphi_{n}\left(\sigma_{n}(s)\right), \varphi_{m}\left(\sigma_{m}(s)\right)\right)(\eta, \xi),
$$

then we have

$$
\begin{aligned}
& \left|\varphi_{n}\left(\sigma_{n}(t)\right)(\eta, \xi)-\varphi_{m}\left(\sigma_{m}(t)\right)(\eta, \xi)\right| \\
\leq & \left|\int_{t_{0}}^{\sigma_{n}(t)} p_{n, n}(s)(\eta, \xi) d s-\int_{t_{0}}^{\sigma_{m}(t)} p_{m, m}(s)(\eta, \xi) d s\right| \\
+ & \int_{t_{0}}^{\sigma_{n}(t)}\left|g_{n}(s)(\eta, \xi)\right| d s+\int_{t_{0}}^{\sigma_{m}(t)}\left|g_{m}(s)(\eta, \xi)\right| d s \\
\leq & \int_{\sigma_{n}(t)}^{t_{0}}\left|p_{n, n}(s)(\eta, \xi)\right| d s-\int_{\sigma_{m}(t)}^{t_{0}}\left|p_{m, m}(s)(\eta, \xi)\right| d s \\
+ & \int_{t_{0}}^{T}\left|p_{n, n}(s)(\eta, \xi)-p_{m, m}(s)(\eta, \xi)\right| d s+\int_{t_{0}}^{\sigma_{n}(t)}\left|g_{n}(s)(\eta, \xi)\right| d s \\
+ & \int_{t_{0}}^{\sigma_{m}(t)}\left|g_{m}(s)(\eta, \xi) d s\right|
\end{aligned}
$$

From $(i)$ and $(i i)$ in Theorem (5.1) we have both $t-\sigma(t) \leq \epsilon_{n}$ and $\left|g_{\eta \xi, n}(s)\right| \leq \epsilon_{n}$.

Also, from definition $\left(Q_{3}\right)$, we have

$$
\left|p_{n, n}(s)(\eta, \xi)-p_{m, m}(s)(\eta, \xi)\right| \leq k_{\eta, \xi}^{\mathcal{P}}\left\|\varphi_{n}\left(\sigma_{n}(t)\right)-\varphi_{m}\left(\sigma_{m}(t)\right)\right\|_{\eta, \xi}
$$

for each $n, m \in N$, from the inequality above and (5.2) we obtain

$$
\begin{aligned}
& \left|\varphi_{n}\left(\sigma_{n}(t)\right)(\eta, \xi)-\varphi_{m}\left(\sigma_{m}(t)\right)(\eta, \xi)\right| \\
& \leq\left(T-t_{0}+M\right)\left(\epsilon_{n}+\epsilon_{m}\right)+k_{\eta, \xi}^{\mathcal{P}} \int_{t_{0}}^{t}\left\|\varphi_{n}\left(\sigma_{n}(s)\right)-\varphi_{m}\left(\sigma_{m}(s)\right)\right\|_{\eta, \xi} d s \\
& +\int_{t_{0}}^{T}\left|p_{n, n}(s)(\eta, \xi)-p_{m, m}(s)(\eta, \xi)\right| d s
\end{aligned}
$$


Using the above inequality and Gronwall's Lemma we have

$$
\left.\begin{array}{l}
\left|\varphi_{n}\left(\sigma_{n}(t)\right)(\eta, \xi)-\varphi_{m}\left(\sigma_{m}(t)\right)(\eta, \xi)\right| \leq\left[\left(T-t_{0}+M\right)\left(\epsilon_{n}+\epsilon_{m}\right)\right. \\
\left.+\int_{t_{0}}^{T}\left|p_{n, n}(s)(\eta, \xi)-p_{m, m}(s)(\eta, \xi)\right| d s\right] e^{k^{P_{\eta, \xi}\left(T-t_{0}\right)}}
\end{array}\right\}
$$

We complete the proof with the following compactness lemma

Lemma 5.4. By the hypothesis of Theorem 5.3, for each subsequence of approximate solutions of equation (3.3), $\left(\sigma_{n}, g_{n, \eta \xi}, \varphi_{n, \eta \xi}\right)_{n}$, there exists one subsequence of $\left(\varphi_{n, \eta \xi}\right)_{n}$, denoted again by $\left(\varphi_{n, \eta \xi}\right)_{n}$, such that for each $\epsilon>0$ there exists $k(\epsilon) \in N$ such that, for each $k, p \in N, k, p \geq k(\epsilon)$ we have

$$
\int_{t_{0}}^{T}\left|\mathcal{P}\left(s, \varphi_{k}\left(\sigma_{k}(s)\right), y(s)\right)(\eta, \xi)-\mathcal{P}\left(s, \varphi_{p}\left(\sigma_{p}(s)\right), y(s)\right)(\eta, \xi)\right| d s \leq \epsilon
$$

uniformly for all maps $y:\left[t_{0}, T\right] \longrightarrow D_{\eta \xi}\left(x_{0}, \rho\right) \cap K$.

This makes the map

$s \longrightarrow\left|\mathcal{P}\left(s, \varphi_{n}\left(\sigma_{n}(s)\right), y(s)\right)(\eta, \xi)\right|$ integrable for $n \in N$.

Proof. Let $\epsilon>0, \delta(\epsilon)>0$ be given by definition (iii) above and let $S$ be a countable dense subset in $\left[t_{0}, T\right]$ with $t_{0}, T \in S$.

Using definition (i) above we find a subsequence of $\left(\varphi_{n, \eta \xi}\left(\sigma_{n}\right)\right)_{n}$, denoted for simplicity again by $\left(\varphi_{n, \eta \xi}\left(\sigma_{n}\right)\right)_{n}$, such that, for each $\theta \in S,\left(\mathcal{P}\left(s,\left(\varphi_{n}\left(\sigma_{n}(\theta)\right)(\eta, \xi), y_{\eta \xi}\right)_{n}\right.\right.$ is Cauchy, for each $s \in\left[t_{0}, T\right]$ and uniformly with respect to $y_{\eta \xi} \in D\left(x_{0}, \rho\right)(\eta, \xi) \cap K(\eta, \xi)$.

Let $\triangle: t_{0}=t_{1}<t_{2}<\ldots<t_{m(\epsilon)}=T$ be a partition of $\left[t_{0}, T\right]$, with $t_{1} \in S$ for $i=1,2, \ldots m(\epsilon)-1$ and

$$
t_{i+1}-t_{1} \leq \frac{\delta(\epsilon)}{2(M+1)}
$$

where $\delta(\epsilon)$ is given by definition $\left(Q_{4}\right)$. So, for the very same $\epsilon>0$, there exists $k(\epsilon) \in N$ such that, for each $n, m \in N, n, m \geq k(\epsilon)$, each $j=1,2, \ldots m(\epsilon)$ and each $(s, y) \in D\left(x_{0}, \rho\right)(\eta, \xi) \cap K(\eta, \xi)$, we have

$$
\left|\mathcal{P}\left(s, \varphi_{n}\left(\sigma_{n}\left(t_{j}\right)\right), y\right)(\eta, \xi)-\mathcal{P}\left(s, \varphi_{m}\left(\sigma_{m}\left(t_{j}\right)\right), y\right)(\eta, \xi)\right| \leq \epsilon
$$

Now, taking a greater $k(\epsilon)$ if necessary, we may assume that for each $n \in N, n \geq$ $k(\epsilon)$, we have

$$
\epsilon_{n} \leq \frac{\delta(\epsilon)}{4(M+1)}
$$

from $(i)$ and $(i i)$ in Theorem (5.2) and equation (5.2), we have for each $n \in N, n \geq$ $k(\epsilon)$,

$$
\left|\varphi_{n}\left(\sigma_{n}(s)\right)(\eta, \xi)-\varphi_{n}\left(\sigma_{n}\left(t_{i}\right)\right)(\eta, \xi)\right| \leq(M+1)\left|\sigma_{n}(s)-\sigma_{n}\left(t_{i}\right)\right|
$$




$$
\leq(M+1)\left(|\sigma(s)-s|+\left|s-t_{i}\right|+\left|t_{i}-\sigma\left(t_{i}\right)\right|\right) \leq \delta(\epsilon)
$$

for each $i=1 \ldots m(\epsilon)-1$ and each $s \in\left[t_{i}, t_{i+1}\right]$. Accordingly, for each $k, p \in N, k, p \geq$ $k(m, \epsilon)=k(\epsilon)$ and each $y:\left[t_{0}, T\right] \longrightarrow D\left(x_{0}, \rho\right)(\eta, \xi) \cap K(\eta, \xi)$ making the map $s \longrightarrow \mathcal{P}\left(s, \varphi_{n}\left(\sigma_{n}(s)\right), y(s)(\eta, \xi)\right)$ integrable for each $n \in N$, we have

$$
\begin{aligned}
& \int_{t_{0}}^{T}\left|\mathcal{P}\left(s, \varphi_{k}\left(\sigma_{k}(s)\right), y(s)\right)-\mathcal{P}\left(s, \varphi_{p}\left(\sigma_{p}(s)\right), y(s)\right)(\eta, \xi)\right| d s \\
\leq & \sum_{i=1}^{m(\epsilon)-1} \int_{t_{i}}^{t_{i}+1} \mid\left(\mathcal { P } \left(s, \varphi_{k}\left(\sigma_{k}(s)\right), y(s)(\eta, \xi)-\mathcal{P}\left(s, \varphi_{k}\left(\sigma_{k}\left(t_{i}\right)\right), y(s)\right)(\eta, \xi) \mid d s\right.\right. \\
& +\sum_{i=1}^{m(\epsilon)-1} \int_{t_{i}}^{t_{i}+1}\left|\left(\mathcal{P}\left(s, \varphi_{k}\left(\sigma_{k}(s)\right), y(s)(\eta, \xi)\right)-\mathcal{P}\left(s, \varphi_{p}\left(\sigma_{p}\left(t_{i}\right)\right)(\eta, \xi), y_{\eta \xi}(s)\right)\right)\right| d s \\
& +\sum_{i=1}^{m(\epsilon)-1} \int_{t_{i}}^{t_{i}+1} \mid\left(\mathcal{P}\left(s, \varphi_{k}\left(\sigma_{k}(s)\right), y(s)(\eta, \xi)\right)-\mathcal{P}\left(s, \varphi_{p}\left(\sigma_{p}\left(t_{i}\right)\right), y(s)\right)(\eta, \xi) \mid d s\right. \\
\leq & \left(T-t_{0}\right) \epsilon+\left(T-t_{0}\right) \epsilon+\left(T-t_{0}\right) \epsilon=3\left(T-t_{0}\right) \epsilon
\end{aligned}
$$

the result above holds true uniformly for each map $y:\left[t_{0}, T\right] \longrightarrow D\left(x_{0}, \rho\right)(\eta, \xi) \cap$ $K(\eta, \xi)$ with $s \longrightarrow \mathcal{P}\left(s, \varphi_{n}\left(\sigma_{n}(s)\right)(\eta, \xi), y_{\eta \xi}(s)\right)$ integrable for each $p \in N$ and this complete the proof of the Lemma 5.4 .

We now complete the proof of Theorem 5.3 by using Lemma 5.4 and Gronwall's inequality

Using Lemma 5.4, we assume that for each $\epsilon>0$ there exists $n_{0}(\epsilon) \in N$ such that for each $n, m \in N, n, m \geq n_{0}(\epsilon)$, we have

$$
\int_{t_{0}}^{T}\left|\left(p_{n, m}(s)-p_{m, m}(s)\right)(\eta, \xi)\right| d s \leq \epsilon
$$

Moreover, since $\lim _{n \rightarrow \infty} \epsilon=0$, for the same $\epsilon>0$, there exists $n_{1}(\epsilon)$ such that, for each $n, m \in N, n, m \geq n_{1}(\epsilon)$, we have

$$
\left(T-t_{0}+M\right)\left(\epsilon_{n}+\epsilon_{m}\right) \leq \epsilon
$$

So, if $n, m \in N, n, m \geq n(\epsilon)=\max \left\{n_{0}(\epsilon), n_{1}(\epsilon)\right\}$, then from (4.12) we conclude

$$
\left|\left(\varphi_{n}\left(\sigma_{n}(t)\right)(\eta, \xi)-\varphi_{m}\left(\sigma_{m}(t)\right)\right)(\eta, \xi)\right| \leq 2 \epsilon e^{k_{\eta \xi}^{\mathcal{P}}\left(T-t_{0}\right)}
$$

which means that $\left(\varphi_{n, \eta \xi}\left(\sigma_{n}(.)\right)\right)_{n}$ is a sequence with respect to the seminorm. Therefore there exists

$$
|\varphi(t)(\eta, \xi)|=\lim _{n \rightarrow \infty}\left|\varphi_{n}\left(\sigma_{n}(t)\right)(\eta, \xi)\right|
$$


uniformly for $t \in\left[t_{0}, T\right]$ which, in view of $(i)$ in Theorem (5.1) and from the continuity of $\mathbb{P}$, there exist successively

$$
\lim _{n \rightarrow \infty}\left|\varphi_{n}(s)(\eta, \xi)\right|=|\varphi(s)(\eta, \xi)|
$$

and

$$
\lim _{n \rightarrow \infty}\left|P\left(s, \varphi_{n}\left(\sigma_{n}(s)\right)\right)(\eta, \xi)\right|=\mid P(s, \varphi(s)(\eta, \xi) \mid
$$

for $s \in\left[t_{0}, T\right]$. So, using the limit $n \rightarrow \infty$ in (iv) of Theorem (5.2) to obtain

$$
\left|\varphi_{\eta \xi}(t)\right| \in\left|x_{0, \eta \xi}\right|+\int_{t_{0}}^{t}|P(s, \varphi(s))(\eta, \xi)| d s
$$

and $\varphi_{\eta \xi}(t) \in D\left(x_{0}, \rho\right)(\eta, \xi) \cap K(\eta, \xi)$ for each $s \in\left[t_{0}, T\right]$. Thus $\varphi_{\eta \xi}$ is a solution of (4.3) on $\left[t_{0}, T\right]$ satisfying

$$
\left|\varphi_{\eta \xi}\left(t_{0}\right)\right|=\left|x_{0, \eta \xi}\right|
$$

and this completes the proof.

\section{REFERENCES}

[1] J.P. Aubin and A. Cellina, Differential Inclusions, Springer Verlag, New York, 1984.

[2] J.P. Aubin, Viability Theory, Birkhauser, Berlin, 1991.

[3] J.P. Aubin, A.M. Bayen and P.S. Pierre, Viability Theory New Directions, Springer- Verlag, Berlin, 2011.

[4] E.O. Ayoola, On convergence of linear multi-step schemes for weak solutions of quantum stochastic differential equations Acta Applicandae mathematicae, 67:19-58, 2001.

[5] E.O. Ayoola, Lipschitzian quantum stochastic differential equations and the associated Kurzweil equations problems, Stochastic Analysis and Applications, 19:4, 581-603, 2001.

[6] E.O. Ayoola, Error estimates for discretized quantum stochastic differential inclusions, Stochastic Analysis and Applications, 21:6, 1215-1230, 2003.

[7] E.O. Ayoola, Exponential formula for the reachable sets of quantum stochastic differential inclusions, Stochastic Analysis and Applications, 21:3, 515-1543, 2003.

[8] E.O. Ayoola, Continuous selections of solution sets of Lipschitzian quantum stochastic differential inclusions, International Journal of Theoretical Physics, 43:10, 2041-2059, 2004.

[9] E.O. Ayoola, Topological properties of solution sets of Lipschitzian quantum stochastic differential inclusions, Acta Applicandae Mathematical, 100, 15-37, 2008.

[10] G.O.S. Ekhaguere, The functional Ito formula in quantum stochastic calculus, Journal of Mathematical Physics, 31:12, 2921-2929, 1990.

[11] G.O.S. Ekhaguere, Lipschitzian quantum stochastic differential inclusions, International Journal of Theoretical Physics, 31:11, 2003-2034, 1992.

[12] G.O.S. Ekhaguere, Topological solutions of Non commutative stochastic differential equations, Stochastic Analysis and Applications, 25:9, 961-993, 2007.

[13] G.O.S. Ekhaguere, Quantum stochastic differential inclusions of hypermaximal monotone type , International Journal of Theoretical Physics , 34:3, 323-353, 1995.

[14] G.O.S. Ekhaguere, Quantum stochastic evolutions, International Journal of Theoretical Physics , 35:9, 1909-1946, 1996.

[15] R.L. Hudson and K.R. Parthasarathy Quantum Ito's formula and stochastic evolutions, Communications in Mathematical Physics, 93:3, 301-323, 1984. 
[16] C. Ovidiu and D. Manuel, Viability results for nonautonomous differential inclusions, Journal of Convex Analysis, 7:2, 437-443, 2000.

[17] C. Ovidiu and V. Ioan, Differential equations on Closed Sets,Handbook of differential equations volume II , Elsevier, North Holland, 2005.

[18] C. Ovidiu, N. Mihai and V. Ioan Viability, Invariance and Applications, Elsevier, North Holland, 2006

[19] G.V. Smirnov, Introduction to the Theory of Differential Inclusions, SISSA Lecture notes, 1995.

[20] I. Vrabie, A Nagumo type viability theorem, Analele Stiintifice Ale Universitatii, 2005. 